\title{
Near-field resonant energy transfer between spherical quantum dots
}

\author{
Filipa C. R. Peres, Mikhail I. Vasilevskiy \\ Centro de Física, Universidade do Minho, Campus de Gualtar 4710-057 Braga, Portugal
}

\begin{abstract}
Non-radiative (Förster-type) energy transfer of an exciton between two quantum dots (QDs) plays an important role in artificial structures where semiconductor nanocrystal QDs play the role of building blocks. We theoretically study the effect of surrounding medium (e.g. dielectric substrate) on the transfer rate. Applying a simple model to describe the QDs, we demonstrate that the transfer rate can be strongly enhanced in the vicinity of a metal surface if the donor QD is excited in resonance with surface plasmons characteristic of this surface. Then the scaling law with the interdot distance becomes more complex than $R^{-6}$ and the characteristic Förster radius can increase by an order of magnitude. We also show that transfer rate between two QDs is not exactly $\propto R^{-6}$ even within the dipole-dipole approximation, in free space if the electron and hole in the dot are in the weak confinement regime.
\end{abstract}

Keywords: quantum dot, exciton, non-radiative energy transfer

\section{INTRODUCTION}

Quantum dots (QDs), crystalline nanoparticles of a semiconductor material, are of interest for applications in optics and photonics owing to their discrete electronic spectra, adjustable by controlling the QD size. QDs have a relatively large absorption cross-section and a narrow emission spectrum, thus, they can be used as markers (characteristic emitters) or nano-antennas for pumping other species that are hard to excite directly ${ }^{1}$ the process usually called fluorescence resonant energy transfer (FRET). FRET is an important energy transport mechanism at the nanoscale, first proposed by Förster for molecules more than sixty years ago. ${ }^{2}$ As far as semiconductor quantum dots are concerned, this type of energy transfer was first demonstrated by Kagan et al. in specially designed films containing two different sizes of QDs acting as donors and acceptors, respectively. ${ }^{3}$ Later it was shown in a number of works ${ }^{4-7}$ performed on systems composed of two different QD species that the luminescence of the smaller dots (donors) is quenched by the large dots (acceptors), whose emission in turn is enhanced. In some studies, near-field energy transfer in systems of nominally monosize QDs has also been observed. ${ }^{8,9}$ These works revealed that FRET between QDs, as well as in molecular systems, strongly depends on the interparticle distance, emission donor and acceptor concentrations, the geometry of the system and the dielectric environment.

Surprisingly, relatively little work on the theoretical side of the QD FRET phenomena has been done so far. In the article by Scholes and Andrews, ${ }^{10}$ the Förster formula for the dipole-dipole transition rate in the near-field regime was rederived and discussed. It was concluded that the calculated transfer rate between two CdSe QDs (4 nm in diameter) is an order of magnitude below the value extracted from the experiment, ${ }^{4}$ however, the magnitude of the rate is strongly influenced by the Stokes shift between the absorption and emission spectra of the dot and their homogeneous broadening. In a more recent work dedicated to multiple QD structures ${ }^{11}$ it is argued that, while the Förster mechanism dominates the transfer rate at short distances $(R<10 \mathrm{~nm})$, the photon-exchange transfer (with the rate decaying as $\propto R^{-2}$ ) becomes more important in ensembles of QDs distributed over a wide range. This is a question of terminology, of course, since the same dipole-dipole interaction decreases as $R^{-3}$ in the near field and as $R^{-1}$ in the far field regions, accordingly, the transfer rate scales as $R^{-6}$ and $R^{-2}$, respectively. ${ }^{10,12}$ Nevertheless, we note that it is the common opinion in the literature that the Förster energy transfer mechanism between QDs can occur only at very short distances (below $10 \mathrm{~nm}$ ). Is it always so?

Further author information:

F. Peres: E-mail: a62448@alunos.uminho.pt 
In this work we use a simple model of spherical QD with infinite potential barriers at the surface in order to study factors that can enhance the FRET probability between two QDs. We show that the transfer rate increases in the vicinity of an interface between two dielectrics and, in particular, can be enhanced owing to a resonance with surface plasmons if a metallic substrate is used. For example, for CdTe QDs on platinum substrate, the characteristic Förster radius can increase beyond $100 \mathrm{~nm}$ (for two identical dots). Another result obtained in this work concerns the $R$ dependence of the near-field dipole-dipole transfer rate at very short center-to-center distances between the dots. We show that this is not exactly $\propto R^{-6}$ if the transient electron-hole dipole is not stuck to the geometrical center of the QD, which takes place in the weak confinement regime.

\section{MODEL}

\subsection{QD exciton states}

First, we present our model used to calculate the exiton states in a spherical QD of radius $a$. For the sake of simplicity, we shall assume simple parabolic bands and infinite barriers on the surface for both electrons and holes. ${ }^{13}$ Within this model and neglecting the Coulomb interaction between the electron and hole (strong confinement limit, $a<<a_{e x}, a_{e x}$ is the bulk exciton Bohr radius) and the multiple sub-band structure of the valence band, the QD exciton spectrum is given by ${ }^{14}$

$$
E_{n l}=E_{g}+\frac{\hbar^{2} \xi_{n l}^{2}}{2 \mu a^{2}}
$$

where $l=0,1,2, \ldots, n=1,2, \ldots, E_{g}$ is the bulk band gap energy, $\mu$ is the electron-hole reduced mass and $\xi_{n l}$ denotes the $n$-th root of the spherical Bessel function, $j_{l}$ (for example, $\xi_{10}=\pi, \xi_{11} \approx 4.49, \xi_{12} \approx 5.76$, $\left.\xi_{20}=2 \pi, \ldots\right) .{ }^{*}$ Even though the Coulomb interaction is neglected, such electron-hole pairs are traditionally called excitons. The electron and hole envelope functions are the same and given by

$$
\Psi_{n l m}(\mathbf{r})=C_{n l} j_{l}\left(\xi_{n l} r / a\right) \Theta(a-r) Y_{l m}(\theta, \phi),
$$

where $C_{n l}$ is a normalisation constant, $\Theta$ is the Heaviside function, $Y_{l m}$ is a spherical harmonic and $m=-l, \ldots, l$. So, the exciton states can be labelled by the quantum numbers $(n, l, m)$ and they are two-fold degenerate with respect to spin.

Within this model, the dipole moment matrix element for transitions between the exciton vacuum and any of the states (1), $\mathbf{d}^{0 \leftrightarrow n l m}$, has an arbitrary direction and its modulus is simply equal to $d_{c v}$, the transition dipole moment matrix element between valence and conduction bands of the underlying bulk semiconductor,

$$
d_{c v}=\frac{e \hbar}{i m_{0} E_{g}} p_{c v},
$$

where $m_{0}$ is the free electron mass and $2 p_{c v}^{2} / m_{0} \approx 20 \mathrm{eV} \cdot{ }^{15}$ The inverse radiative lifetime, i.e. the transition probability from an excited state to the exciton vacuum, can be expressed as

$$
\tau_{n l m}^{-1}=\omega_{n l} \frac{2 \alpha_{F}}{3 m_{0} c^{2}}\left(\frac{2 p_{c v}^{2}}{m_{0}}\right)\left(\frac{E_{n l}}{E_{g}}\right)^{2}
$$

where $\omega_{n l}=E_{n l} / \hbar$ and $\alpha_{F}$ is the fine structure constant.

In the opposite case of weak confinement regime, $a>>a_{e x}$, the exciton is confined as a whole, with the same envelope function (2), $\Psi_{N L M}\left(\mathbf{R}_{C M}\right)$, where the argument is the radius vector of the centre of mass (CM) of the exciton and the numbers $(N, L, M)$ refer to the CM motion. It has to be multiplied by the hydrogen-type wavefunction representing the relative electron-hole motion, $\psi\left(\mathbf{r}_{e}-\mathbf{r}_{h}\right)$. The lowest transition energy is

$$
E_{10}=E_{g}-R_{e x}+\frac{\hbar^{2} \pi^{2}}{2 M_{e x} a^{2}}
$$

where $M_{e x}$ and $R_{e x}$ are the exciton mass and Rydberg, respectively. Here only the states with $L=M=0$ and $s$-type $\psi\left(\mathbf{r}_{e}-\mathbf{r}_{h}\right)$ are dipole-allowed.

${ }^{*}$ We use this model for the sake of simplicity. Generalization to the more realistic one ${ }^{15}$ taking into account the complex valence band structure of the underlying material and the fine structure of the confined hole states is straightforward. 


\subsection{QD polarizability and absorption cross-section}

We now use the model presented in Sec. 2.1 for the case of strong confinement regime to determine the polarizability and the absorption cross-section of a QD placed in a non-stationary electric field, $\mathbf{E}(t)=\frac{1}{2}\left\{\mathbf{E}_{0} e^{-i \omega t}+\mathbf{E}_{0}^{*} e^{+i \omega t}\right\}$, of electromagnetic radiation, which produces a perturbation described by the Hamiltonian:

$$
\hat{H}_{P}=-\mathbf{d} \cdot \mathbf{E} .
$$

The non-stationary envelope wave function of the exciton can be written as a linear combination of the exciton states:

$$
\Psi(t)=\sum_{n, l, m} c_{n l m}(t) e^{-i E_{n l} t / \hbar} \Psi_{n l m}(\mathbf{r}) .
$$

Substituting (4) into the time dependent Schrödinger equation we obtain:

$$
i \hbar \dot{c}_{n l m}(t)=-e^{+i E_{n l} t / \hbar}\left\langle\Psi_{n l m}|\mathbf{d}| 0\right\rangle \mathbf{E}(t),
$$

where $|0\rangle$ is the exciton vacuum state. Solving these equations by Fourier transformation we obtain the coefficients $c_{n l m}$ :

$$
c_{n l m}(t)=-\frac{\left\langle\Psi_{n l m}|\mathbf{d}| 0\right\rangle}{2}\left\{\frac{e^{-i\left(\hbar \omega-E_{n l}\right) t / \hbar}}{\hbar \omega-E_{n l}} \mathbf{E}(0)+\frac{e^{-i\left(-\hbar \omega-E_{n l}\right) t / \hbar}}{-\hbar \omega-E_{n l}} \mathbf{E}^{*}(0)\right\} .
$$

The polarization energy can be written as the average over a period of the internal product of the electric field and the average dipole moment of the non-stationary state $\Psi(t), E=-\langle\mathbf{E}(t) \cdot \mathbf{p}(t)\rangle_{T}, \mathbf{p}(t)=\langle\Psi(t)|\mathbf{d}| \Psi(t)\rangle$ is the average dipole moment of the dot in the non-stationary state and \langle\rangle$_{T}$ represents the average over a period of the field. On the other hand, the polarization energy can be written as $E=-\frac{1}{4} \chi(\omega)|\mathbf{E}(0)|^{2} V$, where $\chi(\omega)$ is the susceptibility of the QD and $V$ is its volume. Thus, the susceptibility is given by

$$
\chi(\omega)=\frac{4}{V|\mathbf{E}(0)|^{2}}\langle\mathbf{p}(t) \cdot \mathbf{E}(t)\rangle_{T} .
$$

Substituting the matrix elements yields:

$$
\chi(\omega)=-\frac{4\left|\mathbf{d}_{c v} \cdot \mathbf{e}\right|^{2}}{V} \sum_{n, l}(2 l+1)\left\{\frac{\hbar \omega-E_{n l}-i \gamma}{\left[\hbar \omega-E_{n l}\right]^{2}+\gamma^{2}}-\frac{\hbar \omega+E_{n l}+i \gamma}{\left[\hbar \omega+E_{n l}\right]^{2}+\gamma^{2}}\right\},
$$

where $\mathbf{e}$ is the unitary polarization vector of the electric field and a small imaginary part, $\gamma>0$, has been prescribed to the energies $E_{n l}$. Performing the average over field polarizations yields $\left|\mathbf{d}_{c v} \cdot \mathbf{e}\right|^{2} \rightarrow \frac{2}{3} d_{c v}^{2}$.

Since the dot can be embedded in a matrix (with dielectric constant $\epsilon_{1}$ ) and the QD material possesses a background dielectric constant $\epsilon_{\infty}^{Q D}$, we need to take into account the depolarization effects because of which the field inside the dot is different from that outside. This is facilitated by introducing the QD polarizability, ${ }^{13}$ which can be written as

$$
\alpha(\omega)=\alpha_{0}+\kappa_{0}^{2} V \frac{\chi}{\epsilon_{1}}
$$

where $\alpha_{0}=a^{3} \frac{\epsilon_{\infty}^{Q D}-\epsilon_{1}}{\epsilon_{\infty}^{Q D}+2 \epsilon_{1}}$ is a frequency-independent background polarizability and $\kappa_{0}=\frac{3 \epsilon_{1}}{\epsilon_{\infty}^{Q D}+2 \epsilon_{1}}$.

The absorption cross-section can be obtained through the imaginary part of the QD polarizability using the relation $^{16} \sigma(\omega)=4 \pi k \operatorname{Im}(\tilde{\alpha})$ with $k=\sqrt{\epsilon_{1}} \omega / c$, from which we have:

$$
\sigma(\omega)=\frac{32 \pi \gamma \kappa_{0}^{2} \omega\left|d_{c v}\right|^{2}}{3 c \sqrt{\epsilon_{1}}} \sum_{n, l}(2 l+1)\left\{\frac{1}{\left[\hbar \omega-E_{n l}\right]^{2}+\gamma^{2}}+\frac{1}{\left[\hbar \omega+E_{n l}\right]^{2}+\gamma^{2}}\right\} .
$$

It is well known that the QD absorption cross-section is large when the photon energy is close to one of the exciton transition energies. From Eq. (8) we can see that it depends on the homogeneous broadening of the exciton states, $\gamma$, which is determined mostly by their interaction with phonons. 


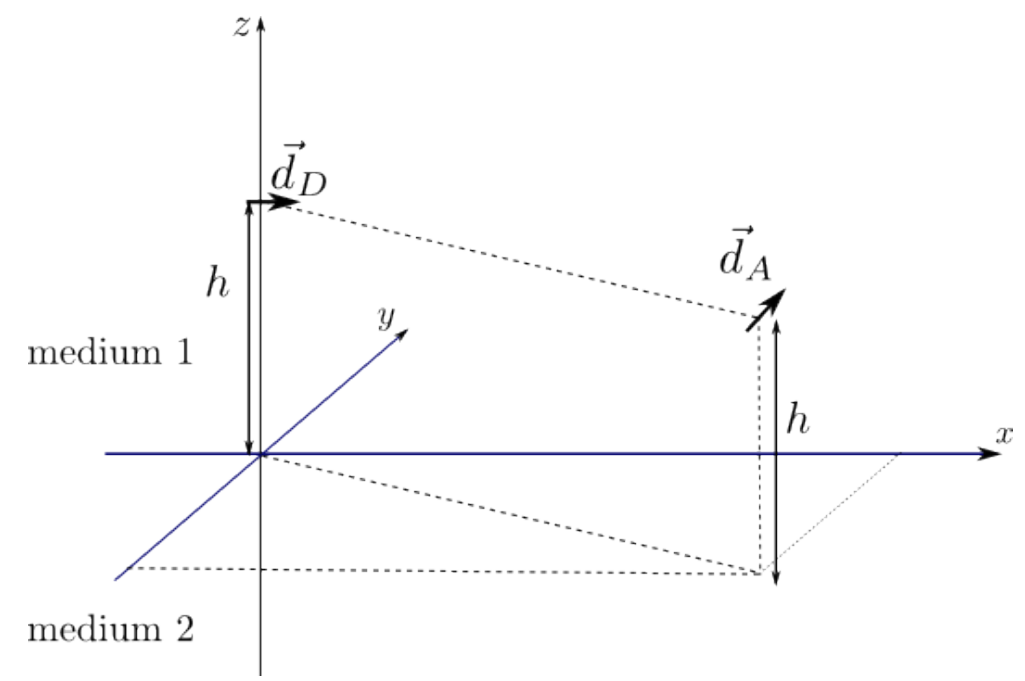

Figure 1. Schematics of the system of two dipoles (polarized QDs) in the vicinity of an interface between two dielectrics.

\subsection{Dipole-dipole interaction between QDs}

Let us now consider the situation where we have two QDs placed at a distance $h$ from the interface $(z=0)$ between two dielectric media (see Fig. 1). We shall assume that both dots (donor and acceptor) are located in the $z=h$ plane, so that the radius vector of the acceptor QD with respect to the donor is $\mathbf{R}=x_{A} \mathbf{e}_{x}+y_{A} \mathbf{e}_{y}$, $x_{A}$ and $y_{A}$ being the $x$ and $y$ coordinates of the former. The donor QD has been excited by a short laser pulse and possesses a transient dipole moment $\mathbf{d}_{D}^{i \rightarrow 0}$, where $i$ stands for the set of quantum numbers $(n, l, m)$ of the excited state of the dot. Now this dipole creates an electromagnetic field which acts on the (initially not excited) acceptor QD. It can be shown ${ }^{10}$ that the FRET matrix element between two QDs can be written in the form resembling the electrostatic interaction between two classical dipoles:

$$
\mathrm{M} \equiv\langle f|\hat{H}| i\rangle=-\mathbf{E}\left(\mathbf{r}_{A}\right) \cdot \mathbf{d}_{A}^{0 \rightarrow f},
$$

where $\mathbf{E}\left(\mathbf{r}_{A}\right)$ is the (transient) electric field created by the donor dot in its state $|i\rangle$ and $\mathbf{d}_{A}^{0 \rightarrow f}$ is the dipole moment matrix element betwen the vacuum and the final exciton state, $|f\rangle$, of the second QD (the upper indices for dipole moments will be omitted for clarity). In the near-field zone $(k R<<1)$, retardation effects can be neglected and in free space we would have $|\mathbf{E}| \propto R^{-3}$. However, the dipole-dipole interaction between the dots is affected by the polarization charges that arise at the interface between two media if their dielectric constants $\left(\epsilon_{1}\right.$ and $\left.\epsilon_{2}\right)$ are unequal. Our purpose here is to calculate the matrix element (9) taking into account the induced surface charges. This problem can be solved in a straightforward manner using the method of images. ${ }^{17}$

The total electric field at $z>0$ is given by the field generated by $\mathbf{d}_{D}$ and that created by an image dipole $\mathbf{d}^{\prime}$ placed in the position $(0,0,-h)$. Thus, the electric field at $z>0$ is:

$$
\mathbf{E}_{z>0}=\frac{3 \mathbf{n}\left(\mathbf{n} \cdot \mathbf{d}_{D}\right)-\mathbf{d}_{D}}{\epsilon_{1} R^{3}}+\frac{3 \mathbf{n}^{\prime}\left(\mathbf{n}^{\prime} \cdot \mathbf{d}^{\prime}\right)-\mathbf{d}^{\prime}}{\epsilon_{1} R^{3}\left(1+\delta^{2}\right)^{3 / 2}},
$$

where $\mathbf{n}=\mathbf{R} / R, \mathbf{n}^{\prime}=\left(\mathbf{n}+\delta \mathbf{e}_{z}\right) / \sqrt{1+\delta^{2}}$ is the unit vector along the direction from the image dipole $\mathbf{d}^{\prime}$ to the acceptor dipole, $\delta=2 h / R$. The electric field at $z<0$ is determined by $\mathbf{d}_{D}$ and a second image dipole, $\mathbf{d}^{\prime \prime}$, and is given by an expression similar to Eq. (10). Using the electrostatic boundary conditions we can determine the image dipoles and thus the electric fields. It is convenient to consider separately two possible orientations of the donor dipole moment, (i) parallel and (ii) perpendicular to the interface. 
In the case (i) we obtain $\mathbf{d}^{\prime}=\mathbf{d}_{D}\left(\epsilon_{1}-\epsilon_{2}\right) /\left(\epsilon_{1}+\epsilon_{2}\right)$ and the matrix element (9) is given by

$$
\mathrm{M}_{\|}=\frac{1}{\epsilon_{1} R^{3}}\left\{\left(\mathbf{d}_{A} \cdot \mathbf{d}_{D}\right)\left(1+\frac{\mathcal{R}}{\left(1+\delta^{2}\right)^{3 / 2}}\right)-3\left(\mathbf{d}_{D} \cdot \mathbf{n}\right)\left(\mathbf{d}_{A} \cdot \mathbf{n}\right)\left(1+\frac{\mathcal{R}}{\left(1+\delta^{2}\right)^{5 / 2}}\right)-\left(\mathbf{d}_{D} \cdot \mathbf{n}\right) d_{A z} \frac{3 \delta \mathcal{R}}{\left[1+\delta^{2}\right]^{5 / 2}}\right\},
$$

where $\mathcal{R}=\frac{\epsilon_{1}-\epsilon_{2}}{\epsilon_{1}+\epsilon_{2}}$ and $d_{A z}$ is the $z$ component of the dipole moment of the acceptor.

In the case (ii) the image dipole is $\mathbf{d}^{\prime}=-\mathbf{d}_{D}\left(\epsilon_{1}-\epsilon_{2}\right) /\left(\epsilon_{1}+\epsilon_{2}\right)$ (notice the opposite sign) and the matrix element is:

$$
\mathrm{M}_{\perp}=\frac{1}{\epsilon_{1} R^{3}}\left\{\left(\mathbf{d}_{A} \cdot \mathbf{d}_{D}\right)\left(1-\frac{\mathcal{R}}{\left(1+\delta^{2}\right)^{3 / 2}}+\frac{3 \delta^{2} \mathcal{R}}{\left(1+\delta^{2}\right)^{5 / 2}}\right)+\left(\mathbf{d}_{A} \cdot \mathbf{n}\right) d_{D} \frac{3 \delta \mathcal{R}}{\left[1+\delta^{2}\right]^{5 / 2}}\right\} .
$$

The square of the matrix element $\mathrm{M}$ averaged over all possible orientations of the acceptor dipole determines the transfer probability. After performing this average we obtain the following expressions:

$$
\begin{array}{r}
\left|\mathrm{M}_{\|}\right|^{2}=\frac{\mathrm{M}_{0}^{2}}{2 \epsilon_{1}^{2}}\left\{\frac{5}{2}+\frac{|\mathcal{R}|^{2}}{2} \frac{5+2 \delta^{2}}{\left(1+\delta^{2}\right)^{4}}+\operatorname{Re}(\mathcal{R}) \frac{5-\delta^{2}}{\left(1+\delta^{2}\right)^{5 / 2}}\right\} \\
\left|\mathrm{M}_{\perp}\right|^{2}=\frac{\mathrm{M}_{0}^{2}}{2 \epsilon_{1}^{2}}\left\{\left|1-\mathcal{R} \frac{1-2 \delta^{2}}{\left(1+\delta^{2}\right)^{5 / 2}}\right|^{2}+\left|\frac{3 \mathcal{R} \delta}{\left(1+\delta^{2}\right)^{5 / 2}}\right|^{2}\right\},
\end{array}
$$

where $\mathrm{M}_{0}^{2}=\frac{2\left|d_{A}\right|^{2}\left|d_{D}\right|^{2}}{3 R^{6}}$. We keep the modulus in the right-hand side of (13) because $\mathcal{R}$ can be complex, e.g. in the case when the medium 2 is a metal.

\section{RESULTS AND DISCUSSION}

\subsection{Transfer probability and resonant enhancement}

The transition probability from an initial state $(n l m)$ of the donor dot, per unit time is given by the Fermi's Golden Rule,

$$
w_{n l m}=\frac{2 \pi}{\hbar} \sum_{n^{\prime}, l^{\prime}, m^{\prime}} 2|\mathrm{M}|^{2} \delta\left(E_{n^{\prime} l^{\prime}}^{A}-E_{n l}^{D}\right)
$$

where the factor of 2 accounts for two possible orientations of spin in the acceptor QD. By virtue of the $\delta$-function, for two identical QDs only the terms with $n^{\prime}=n$ and $l^{\prime}=l$ in (14) have to be kept, so the transition probability increases with $l$ as $(2 l+1)$. Of course, in reality no identical QDs exist but always there is some homogeneous broadening of the exciton energy levels, so the $\delta$-function in (14) may be replaced by a Lorentzian with the width $\gamma$, the same as in Eq. (8). This parameter determines the transition probability and the Förster radius, which is defined as the distance at which the transfer rate becomes equal to the radiative lifetime, ${ }^{2}$ i.e. $\left\langle w_{n l m}\right\rangle=\tau_{n l m}^{-1}$, where the latter is given by Eq. (3) and the transition probability is averaged over the orientations of the donor dipole. ${ }^{\dagger}$

We applied the above formalism to CdTe nanocrystal QDs of average radius $3 \mathrm{~nm}$. This yields a lifetime of $\sim 1 \mathrm{~ns}$ for the lowest exciton state (100), a value which is close to the typical experimental data. ${ }^{1}$ Our simple model slightly overestimates the electron-hole wavefunction's overlap and, consequently, the exciton transition probabilities. Nevertheless, for the Förster radius of transfer between two identical dots in vacuum, with $\gamma=10$ $\mathrm{meV}$ the model gives an optimistic value of $25 \mathrm{~nm}$, significantly more then those usually estimated experimentally. ${ }^{7}$ However, since it involves a free parameter $(\gamma)$ it is better to compare the effect produced by the surrounding dielectrics in terms of the matrix elements (13). They are plotted against the relative distance from the interface, $\delta$, in Fig. 2. From this figure we can see that the effect of the substrate dielectric constant is quite different for the two orientations of the donor dipole. For the dipole along $z$ the matrix element is increased for any value of the dielectric constant if the dots are located in air $\left(\epsilon_{1}=1\right)$. If $\epsilon_{1}>1$ (not shown), the matrix 

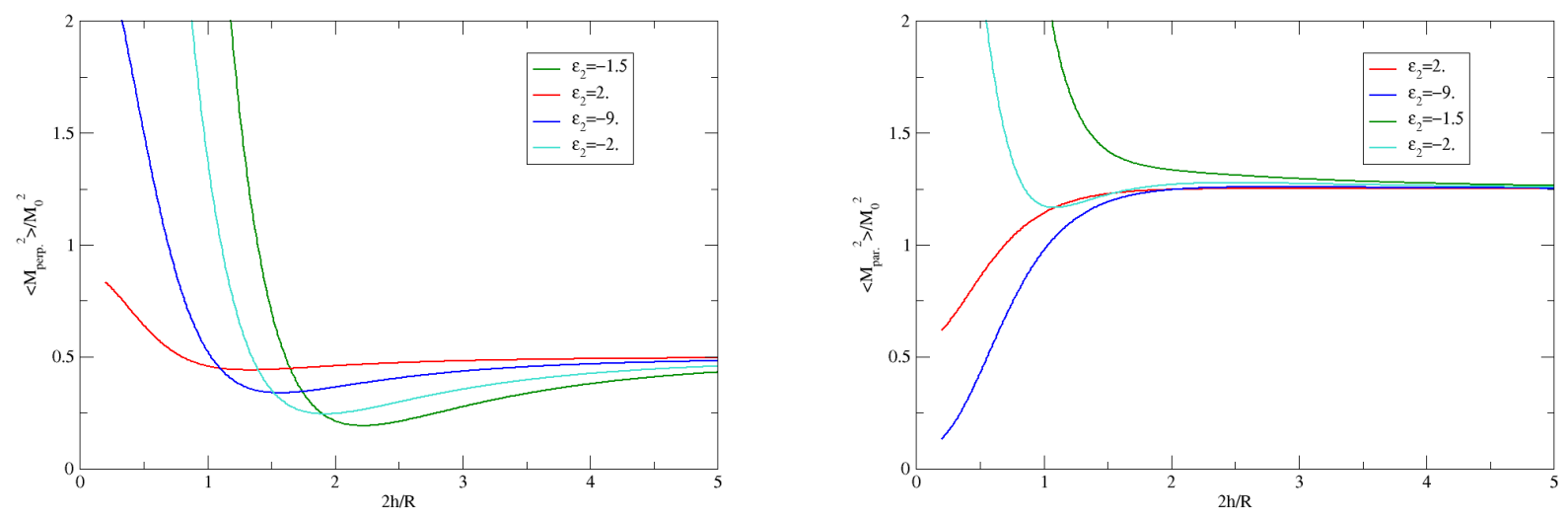

Figure 2. (Colour online) Dependence of the squared transition matrix element on distance from the substrate for donor dipole perpendicular (left) and parallel (right) to the interface, for different values of the dielectric constant of the substrate. $\epsilon_{1}=1$ in all cases.

element is reduced near the interface. For a donor dipole parallel to the interface, the dependence upon the dielectric constant of the substrate $\left(\epsilon_{2}\right)$ is non-monotonic.

The term proportional to $\left(\epsilon_{1}-\epsilon_{2}\right)$ (i.e. to $\mathcal{R}$ ) in the matrix elements, originating from the polarization charges on the interface becomes large if $\left(\epsilon_{1}+\epsilon_{2}\right) \rightarrow 0$. This condition can be fulfilled for a metal/dielectric interface and corresponds to the excitation of surface plasmons (SPs). ${ }^{18}$ We can notice the strong increase of the matrix elements when the substrate dielectric constant approaches the value of -1 . Figure 3 illustrates better this SP resonance for the case of QDs embedded in a dielectric matrix with $\epsilon_{1}>1$ and placed on a platinum substrate. $\ddagger$ Also shown in this figure is the absorption cross-section averaged over an ensemble of QDs with some size dispersion (Gaussian distribution of $a$ ). For the higher-energy states, a large enhancement of the transition probability can be achieved, especially in the case of donor dipole perpendicular to the interface. For this, the SP resonance has to match one of the excitonic resonances in the QDs. It can be achieved by the appropriate choice of the dielectric constant of the medium where the dots are immersed and/or by adjusting their mean size. If this is achieved, the transition probability can be so high that FRET may become the dominant de-excitation channel even for high-energy exciton states (which usually do not emit light because the non-radiative relaxation processes are faster than the radiative lifetime $\tau$ ).

\subsection{Dependence on the interdot distance}

First, we would like to point out that the electromagnetic interaction between two "identical" QDs is restricted to the dipole-dipole one if the donor dot was excited via a dipole-allowed transition. Then, if the QDs are in the strong confinement regime, their interaction must scale with the inter-dot distance as $R^{-3}$ in free space (and the FRET probability as $R^{-6}$ ). Of course, near an interface of two media with $\epsilon_{1} \neq \epsilon_{2}$ the scaling law is more complex - see Fig. 4 (left).

However, deviations from the $R^{-3}$ dependence appear already for free space in the weak confinement regime, where the exciton (and, consequently, the transient dipole moment) is not stuck to the center of the dot. Considering the interaction of two such dipoles, each confined to a sphere of radius $a$, leads to the near-field transition matrix element of the form:

$$
\mathrm{M}=\left|\psi_{s}(0)\right|^{2}\left|\mathbf{d}_{c v}\right|^{2} \int \Psi_{N 00}\left(\mathbf{R}_{1}\right) \Psi_{N 00}\left(\mathbf{R}_{2}\right) \frac{\left(\mathbf{e}_{1} \cdot \mathbf{e}_{2}\right)-3\left(\mathbf{n} \cdot \mathbf{e}_{1}\right)\left(\mathbf{n} \cdot \mathbf{e}_{2}\right)}{R_{12}^{3}} d^{3} \mathbf{R}_{1} d^{3} \mathbf{R}_{2},
$$

\footnotetext{
${ }^{\dagger}$ It means that we should substitute into (14) $|\mathrm{M}|^{2}=\frac{1}{3}\left|\mathrm{M}_{\perp}\right|^{2}+\frac{2}{3}\left|\mathrm{M}_{\|}\right|^{2}$.

${ }^{\ddagger}$ The dielectric constant of Pt was described by the Drude model with the plasma frequency $\hbar \omega_{p}=5.15 \mathrm{eV}$ and damping $\hbar \Gamma_{p}=70 \mathrm{meV}$.
} 

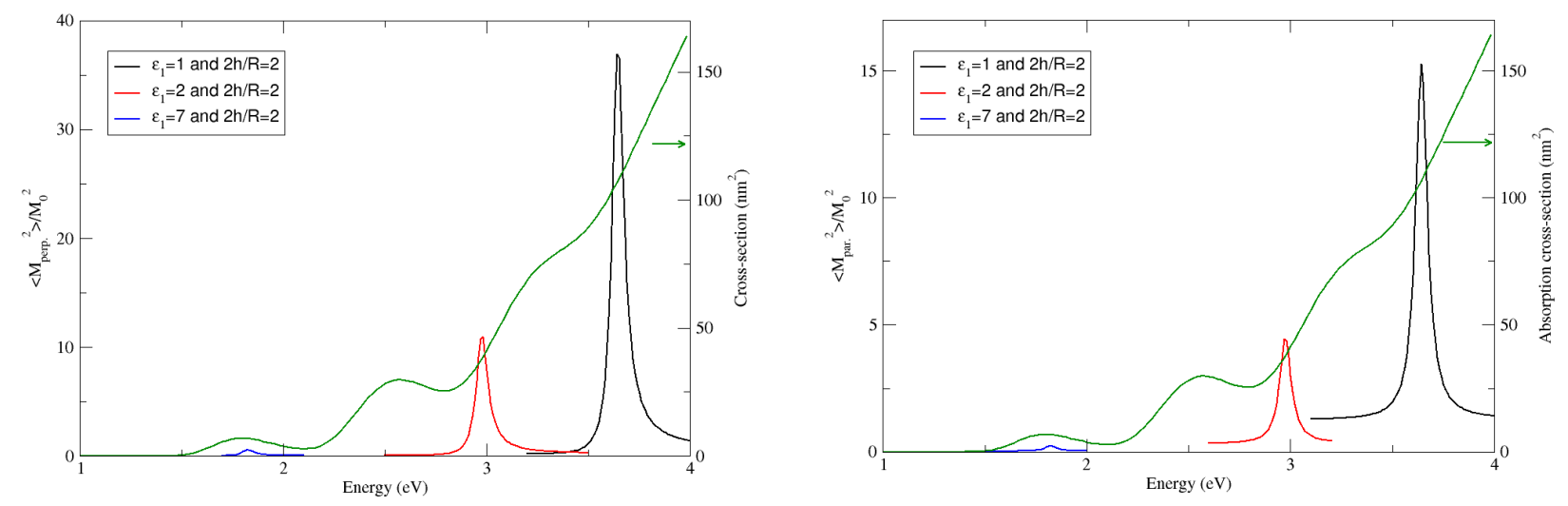

Figure 3. Spectral dependence of the enhancement factor in the vicinity of a plamonic substrate, for donor dipole perpendicular (left) and parallel (right) to the interface, for different values of the dielectric constant of the medium where QDs are embedded. Also shown is the average absorption cross-section of an ensemble of CdTe QDs with $a=(3 \pm 0.25) \mathrm{nm}$. Other parameters are: $\gamma=10 \mathrm{meV}, \kappa_{0}=1$.

where $\mathbf{e}_{1}$ and $\mathbf{e}_{2}$ the unit vectors along the dipole moments $\mathbf{d}_{D}$ and $\mathbf{d}_{A}$, respectively, $\mathbf{R}_{12}=\mathbf{R}_{2}-\mathbf{R}_{1}$ and $\mathbf{n}=\mathbf{R}_{12} / R_{12}$. Averaging the square of the numerator in (15) over dipole's orientations will give a numerical factor of $2 / 3$, so we are left with the calculation of the following integral:

$$
I(R)=\int_{Q D} \Psi_{N 00}\left(\mathbf{R}_{1}\right) \Psi_{N 00}\left(\mathbf{R}_{2}\right) \frac{1}{R_{12}^{3}} d^{3} \mathbf{R}_{1} d^{3} \mathbf{R}_{2} .
$$

The calculation of this integral is greatly facilitated if we approximate the center-of-mass wavefunction (given by Eq. (2)) by $\Psi_{100}\left(\mathbf{R}_{C M}\right) \approx \frac{1}{\sqrt{V}} \Theta\left(a-R_{C M}\right)$, then (16) can be reduced to

$$
I(R)=\frac{1}{R^{3}} \int_{Q D} d V\left\{1+\left(\frac{r_{12}}{R}\right)^{2}+\frac{2}{R}\left[r_{2} \cos \left(\theta_{2}\right)-r_{1} \cos \left(\theta_{1}\right)\right]\right\}^{-3 / 2},
$$

where $\mathbf{r}_{12}=\mathbf{r}_{2}-\mathbf{r}_{1}$ and $\mathbf{r}_{1,2}$ are two points within the same sphere of radius $a$. Then $I(R)$ can be evaluated using the Monte-Carlo method by generating random points in pairs. The result is presented in Fig. 4 and shows that the dependence of the dipole-dipole interaction indeed deviates from the standard $R^{-3}$ law at very short distances between the dots. It happens due to the fact that the excitons confined in the different dots can come closer to each other than the center-to-center interdot distance if they are in the weak confinement regime.

\section{CONCLUSION}

In summary, we have shown that the Förster transfer rate between quantum dots is strongly influenced by their dielectric surrounding. In particular, it can be strongly enhanced in the vicinity of a metal7dielectric interface if the donor QD is excited in resonance with the surface plasmons characteristic of this interface. Then the characteristic Förster radius can increase by an order of magnitude. This opens the possibilty to greatly enhance the exciton transport via FRET in QD ensembles, which can have interesting applications in solar energy concentrators. To make this feasible, it is necessary to find appropriate plasmonic substrate and dielectric matrix in order to push the SP resonance to the spectral range of interest. Moreover, with the broad choice of colloidal QDs available, ${ }^{1}$ fine tuning into different exciton resonances could be achieved, thus allowing for interesting experiments aimed at comparing the de-excitation rates of low and high energy exciton states in QDs. Finally, we also have shown that the FRET rate between two QDs where the excitons are in the weak confinement regime, is not exactly $\propto R^{-6}$ even within the dipole-dipole approximation because the exciton-exciton separation can be 

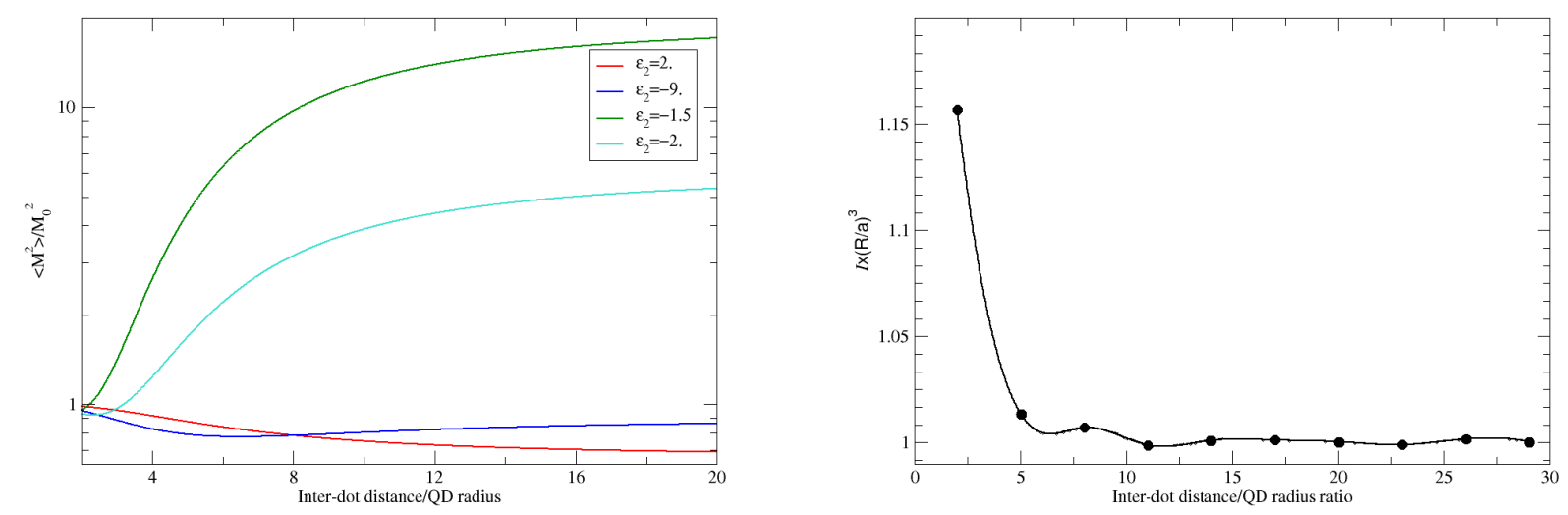

Figure 4. Dependence of the transition matrix element between two QDs upon the center-to-center distance, in the strong confinement regime, near an interface $(h=2 a)$ between vacuum and a dielectric with $\epsilon_{2}$ as indicated (left), and in the weak confinement regime, in free space (right).

smaller than the center-to-center interdot distance. This is different from strongly localized molecular excitations in "traditional" FRET systems.

\section{ACKNOWLEDGMENTS}

Financial support from the Portuguese Foundation for Science and Technology (FCT) through Projects PTDCFIS-113199-2009 and PEst-C/FIS/UI0607/2013 is gratefully acknowledged. FCRP thanks the FCT for BI fellowship.

\section{REFERENCES}

[1] A. L. Rogach (Ed), Semiconductor Nanocrystal Quantum Dots: Synthesis, Assembly, Spectroscopy and Applications, Springer (2008).

[2] T. Förster, Ann. Phys. 437, 55 (1948).

[3] C. R. Kagan, C. B. Murray, M. Nirmal, and M. G. Bawendi, Phys. Rev. Lett. 76, 1517 (1996).

[4] S. A. Crooker, J. A. Hollingsworth, S. Tretiak, and V. I. Klimov, Phys. Rev. Lett. 89, 186802 (2002).

[5] T. Franzl et al., Small 1, 392 (2005).

[6] L. Guo et al., Appl. Phys. Lett. 89, 061104 (2006).

[7] M. Lunz et al., Phys. Rev. B 81, 205316 (2010); Phys. Rev. B 83, 115423 (2011).

[8] A. Sukhanova, A. V. Baranov, T. S. Perova, J. H. Cohen, and I. Nabiev, Angew. Chem. 45, 2048 (2006).

[9] C. Bernardo et al., J. Phys. Chem. C 118, 4982 (2014).

[10] G. D. Scholes and D. L. Andrews, Phys. Rev. B 72, 125331 (2005).

[11] S. K. Lyo, Phys. Rev. B 79, 125328 (2009).

[12] D. L. Andrews and B. S. Sherborne, J. Chem. Phys. 86, 4011 (1987).

[13] Yu. V. Bludov and M. I. Vasilevskiy, J. Phys. Chem. C 116, 13738 (2012).

[14] L. E. Brus, J. Chem. Phys. 80, 4403 (1984).

[15] Al. L. Efros, Phys. Rev. B 46, 7448 (1992); Al. L. Efros et al., Phys. Rev. B 54, 4843 (1996).

[16] M. Born and E. Wolf, Principles of Optics, Pergamon (1989).

[17] J. D. Jackson, Classical Electrodynamics, J. Wiley (1975).

[18] M. G. Cottam and D. R. Tilley, Introduction to Surface and Superlattice Excitations, Cambridge University Press (1989). 